\title{
ON A COVERING SURFACE OVER AN ABSTRACT RIEMANN SURFACE
}

\author{
MAKOTO OHTSUKA*
}

1. Let $\Re$ be an abstract Riemann surface in the sense of Weyl-Radó, and $\Re$ an open covering surface over $\mathfrak{R}$. If a curve $C=\{P(t) ; 0 \leqq t<1\}$ on $\Re$ tends to the ideal boundary of $\mathfrak{R}$ but its projection terminates at an inner point of $\Re$ as $t \rightarrow 1$, we shall say that $C$ determines an accessible boundary point (which will be abbreviated by A.B.P.) of $\Re$ relatively to $\mathfrak{R}$. The set of all the A.B.P.s ${ }^{1)}$ of $\Re$ relative to $\Re$ will be called accessible boundary (relative to $\underline{\Re}$ ) and denoted by $\mathfrak{A}(\mathfrak{R})$ or by $\mathfrak{A}(\mathfrak{R}, \mathfrak{R})$. Throughout in this paper $\mathfrak{A}(\mathfrak{R})$ will be supposed to be non-empty.

After K. I. Virtanen [12] we shall use the notation $\left(B_{0}\right)$ to denote the class of Riemann surfaces, on which no one-valued and non-constant bounded harmonic function exists.

In the first place in this note we shall define harmonic measure $\omega(P)$ of $\mathfrak{Y}(\Re)$ and show that if $\omega(P)>0$ then $\Re \notin\left(B_{0}\right)$.

We suppose next that the projection of $\Re$ is compact in $\mathscr{R}$ and that the universal covering surface $\mathfrak{R}^{\infty}$ of $\mathfrak{R}$ is of hyperbolic type. Then $\mathfrak{R}^{\infty}$ is mapped conformally onto a unit circular domain $U:|z|<1$, and we obtain a function $f(z)$ which maps $U$ into $\mathfrak{R}$, corresponding to the mappings $U \rightarrow \Re^{\infty} \rightarrow \mathfrak{R} \rightarrow \mathfrak{\Re}$. If $f(z)$ tends to a value $f\left(e^{i \theta}\right)$ as $z \rightarrow e^{i \theta}$ along every Stolz's path ${ }^{23)}$ a.e. ( $=$ almost everywhere) on $\Gamma:|z|=1, \mathfrak{R}$ will be called of F-type (relatively to $\mathfrak{R}$ ) (cf. [7], Chap. III, §2).

In $\S 5$ of this note we shall show that $\omega(P) \equiv 1$ for $\Re$ of $F$-type and give a condition so that $\Re$ is of F-type, generalizing a result in [7].

Finally we shall remark some relations between concepts defined in this note.

2. We consider the class $\mathfrak{B}(\Re)$ of all the non-negative continuous super-

Received November 7, 1951.

* This work was done by the writer as a fellow of the Yukawa Foundation of Osaka Uinversity.

1) Any equivalency of A.B.P.s is not considered here.

9) By a Stolz's path we mean a path which terminates at a point on $\Gamma$ and lies between two chords through the point.

3) When $f(\boldsymbol{z})$ has this property, we shall say that $f(\boldsymbol{z})$ has an angular limit at $e^{i \theta}$ and call $f\left(e^{i \theta}\right)$ the angular limit at $e^{i \theta}$. 
harmonic functions $\{v(P)\}$ on $\Re$ such that $v(P) \leqq 1$ and $\lim v(P)=1$ when $P$ tends to $\Re(\Re)$ along every curve determining an A.B.P. of $\mathfrak{R}$ relative to $\mathfrak{R}$. This class is non-empty, since the constant 1 belongs to it. The lower cover $(=$ infimum at every point) of $\mathfrak{B}(\Re)$ is harmonic on $\mathfrak{R}$ by Perron-Brelot's principle (cf. [7], Chap. $I, \S 1$ ), and will be denoted by $\mu(P, \mathfrak{A}(\mathfrak{R})$ ).

First we suppose that the universal covering surface $\underline{\Re}^{\prime \infty}$ of the projection $\underline{R \prime}^{\prime}$ of $\Re$ into $\mathfrak{R}$ is of hyperbolic type; that is, if $\underline{R}^{\prime}$ is of genus zero it is conformally equivalent to a plane domain with at least three boundary points, if $\underline{R}^{\prime}$ is of genus one it is open, and if the genus is greater than one $\underline{R}^{\prime}$ is required to fulfill no further condition. We define harmonic measure (function) $\omega(P)$ of $\mathfrak{Y}(\mathfrak{R})$ by means of $\mu\left(P, \mathfrak{X}\left(\mathfrak{R}^{\infty}, \mathfrak{R}\right)\right)$, which may be regarded as a onevalued function on $\mathfrak{R}$.

The universal covering surface $\Re^{\infty}$ of $\Re$ is also of hyperbolic type and mapped conformally onto $U:|z|<1$. It can be shown that the images in $U$ of a curve determining an A.B.P. of $\mathfrak{A}$ terminate at points on $\Gamma:|z|=1$, which are equivalent with respect to a Fuchsian group, and that, $f(z)$ denoting mapping function of $U$ into $\stackrel{\Re}{R} f(z)$ has an angular limit at any point $e^{i \theta}$ on $\Gamma$, where an image of a determining curve of an A.B.P. terminates. ${ }^{4)}$ We shall call the set of all the points on $\Gamma$, which correspond to A.B.P.s of $\mathfrak{R}$, the image on $\Gamma$ of $\mathfrak{A}(\mathfrak{R})$.

We will now give

THEOREM 1. Let $\mathfrak{A}$ be an open covering surface over an abstract Riemann

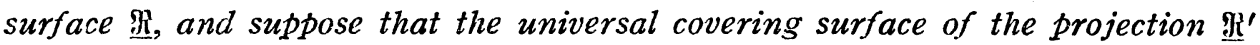
of $\mathfrak{R}$ into $\mathfrak{R}$ is of hyperbolic type. Then the image $E$ on $\Gamma$ of $\mathfrak{A}(\mathfrak{R})$ is linearly measurable and tine value of the harmonic measure $\mu(z, E)$ in $U$ of $E$ is equal to the value of $\mu\left(P, \mathfrak{A}\left(\Re^{\infty}\right)\right)$ at any corresponding points.

Proof. In case $\mathfrak{R}^{\infty}$ is of hyperbolic type, map it conformally onto $U_{w}:|w|$ $<1$. $E$ coincides with the place on $\Gamma$, where any branch of the function corresponding to the mappings $U \rightarrow \mathfrak{R} \rightarrow \mathfrak{R}^{\infty} \rightarrow U_{w}$ has limits lying in $U_{w}$. Namely, $E$ is the complement of the set $E^{\prime}$ on $\Gamma$, where the branch has radial limits on $|w|=1$ or has no limit. Since $E^{\prime}$ is linearly measurable (cf. [7], Chap. IV,§3), $E$ is so too.

In case $\Re^{\infty}$ is of parabolic or elliptic type, map it conformally, onto $|w|<\infty$ or $|w| \leqq \infty$. Since $\mathbb{R}^{\prime \infty}$ is of hyperbolic type, any branch of the function mapping $U$ into the $w$-plane does not take at least three values $w_{1}, w_{2}$ and $w_{3}$. Map further the universal covering surface of the complement of $w_{1}, w_{2}, w_{3}$ onto $U_{\omega}$ : $|\omega|<1$, and let $\omega=F(z)$ be any branch of the function corresponding to the composed mappings. To $w_{1}, w_{2}, w_{3}$ there correspond an enumerably infinite number

4) These results were stated in [7], Chap. III, $\$ 1$ under the assumption that the projection $\underline{\Re}^{\prime}$ is compact in $\Re$.

5) The method in proving the meastrabliity of $E^{\prime}$ is available also to show the measurability of $E$ directly. 
of points $\left\{\omega_{i}\right\}$ on $|\omega|=1 . E$ is classified into the following two parts: $E_{1}$ where $F(z)$ has radial limits lying in $U_{\omega}$, and $E_{2}$, which is a subset of the set $E_{2}^{\prime}$ where the radial limits of $F(z)$ are equal to some of $\left\{\omega_{i}\right\} . E_{2}^{\prime}$ is linearly measurable and its measure is zero by Riesz's theorem [9], and the measurability of $E_{1}$ follows for the same reason as in the first case. Thus $E=E_{1}+E_{2}$ is measurable.

The harmonic measure $\mu(z, E)$ of $E$ is equal to the lower cover of the class $\mathfrak{B}(U)$ consisting of all the non-negative continuous super-harmonic functions $\{v(z)\}$ in $U$, each of which is $\leqq 1$ and tends to 1 as $z$ approaches every point of $E$. If $v(z)$ is considered on $\Re^{\infty}$, it belongs to $\mathfrak{Y}\left(\Re^{\infty}\right)$ and hence

$$
\mu\left(P(z), \mathfrak{A}\left(\mathfrak{R}^{\infty}\right)\right) \leqq \mu(z, E) .
$$

Conversely let $v_{1}(P)$ be any function of $\mathfrak{B}\left(\Re^{\infty}\right)$ and consider it in $U$. Then its radial limit equals 1 at every point of $E$. Letting $\rho \rightarrow 1$ in inequalities

$$
\begin{aligned}
v_{1}(P(z)) & \geqslant \frac{1}{2 \pi} \int_{0}^{2 \pi} v_{1}\left(P\left(\rho e^{i \rho}\right)\right) \frac{\rho^{2}-r^{2}}{\rho^{2}+r^{2}-2 \rho r \cos (\theta-\varphi)} d \varphi \\
& \geqslant \frac{1}{2 \pi} \int_{e^{i \varphi \in E}} v_{1}\left(P\left(\rho e^{i p}\right)\right) \frac{\rho^{2}-r^{2}}{\rho^{2}+r^{2}-2 \rho r \cos (\theta-\varphi)} d \varphi \quad\left(z=r e^{i \theta}, \rho>r\right),
\end{aligned}
$$

we have by Lebesgue's theorem

$$
v_{1}(P(z)) \Rightarrow \frac{1}{2 \pi} \int_{e^{i} \in E E} \frac{1-r^{2}}{1+r^{2}-2 r \cos (\theta-\varphi)} d \varphi=\mu(z, E) .
$$

Consequently we obtain the reverse inequality

$$
\mu\left(P(z), \mathfrak{U}\left(\Re^{\infty}\right)\right) \geqslant \mu(z, E) .
$$

Thus there holds the equality and the theorem is proved.

3. As preparation for the definition of $\omega(P)$ in the case when $\underline{\mathfrak{R}}^{\prime}$ is not of hyperbolic type, we shall prove the following lemma, which will be used also in $\S 5$.

LEMMA. Let the universal covering surface $\mathfrak{R}^{\infty}$ of $\mathfrak{R}$ be of hyperbolic type and map it conformally onto $U$. Suppose that the mapping function $f(z)$ of $U$ into $\mathfrak{R}$ has an angular limit at every point $e^{i \theta}$ belonging to a measurable set $E \subset \Gamma$. Take a finite number of points $\left\{\underline{P}_{i}\right\}(i=1,2, \ldots, n)$ on $\mathfrak{R}$ and remove from $\mathfrak{R}$ all the points lying over them so that the projection of the remaining surface $\widetilde{\mathfrak{R}}$ has a universal covering surface of hyperbolic type.

Then there holds at any corresponding points

$$
\mu(z, E) \leqq \mu\left(P, \mathfrak{A}\left(\widetilde{\mathfrak{H}}^{\infty}\right)\right) .
$$

Proof. Map $\widetilde{\mathfrak{R}}^{\infty}$ onto $U_{\zeta}:|\zeta|<1$ and denote the image on $\Gamma_{\zeta}:|\zeta|=1$ of $\geqq\left(\left(\widetilde{\mathfrak{H}^{\circ}}\right)\right.$ by $E_{\zeta}$. Then by Theorem $1 \mu\left(P, \mathfrak{A}\left(\widetilde{\mathfrak{R}}^{\infty}\right)\right)=\mu\left(\zeta, E_{\zeta}\right)$. Hence we shall show $\mu(z, E) \leqq \mu\left(\zeta, E_{\zeta}\right)$ under the assumption that the linear measure $m(E)>0$. 
Let $E^{\prime}$ be any measurable subset of positive measure of $E$. Any image in $U_{\zeta}$ of a Stolz's path terminating at a point of $E^{\prime}$ terminates at a point of $E_{\zeta}$. We shall call the set of all such end-points on $E_{\zeta}$ the angular image on $E_{\zeta}$ of $E^{\prime}$. In the following we shall show that the angular image on $E_{\zeta}$ of $E^{\prime}$ has a positive linear inner measure.

Consider a non-constant one-valued meromorphic function on $\mathfrak{R}$ and combine it with $f(z)$. The function $F(z)$ thus defined in $U$ is also non-constant one-valued and meromorphic. Let $E^{\prime \prime} \subset E^{\prime}$ be the set where the limits of $f(z)$ are equal to some of $\left\{\underline{P}_{i}\right\}$. Then $F(z)$ has also a finite number of values as its angular limits at points of $E^{\prime \prime} . E^{\prime \prime}$ is measurable and Lusin-Priwaloff's theorem $[2]^{6)}$ shows that the linear measure of $E^{\prime \prime}$ is zero. Hence $m\left(E^{\prime}-E^{\prime \prime}\right)$ $=m\left(E^{\prime}\right)>0$. Denote the angular domain: $\left|\arg \left(1-e^{-i \theta} z\right)\right|<\frac{\pi}{4}$ at $e^{i \theta}$ by $A(\theta)$. By Egoroff's theorem we can find a closed subset $F$ of positive linear measure of $E^{\prime}-E^{\prime \prime}$ such that $f(z)$ tends to the angular limit $f\left(e^{i \theta}\right)$ uniformly as $z \rightarrow e^{i \theta}$ $\in F$ from the inside of $A(\theta)$. In the usual way we get a domain $D \subset U$, which contains an end-part of every $A(\theta)$ for $e^{i \theta} \in F$ and is bounded by a rectifiable curve $C$ consisting of $F$ and segments lying on the boundaries of $\left\{A(\theta) ; e^{i \theta} \in F\right\}$. The number of points $\left\{z_{k}\right\}$ corresponding to $\left\{\underline{P}_{i}\right\}$ and lying on $D+C$ is finite, because $f(z) \rightarrow f\left(e^{i \theta}\right)$ uniformly in $D$ and $\left\{f\left(e^{i \theta}\right) ; e^{i \theta} \in F\right\}$ is a closed set not containing the points $\left\{\underline{P}_{i}\right\}$. By removing $\left\{z_{k}\right\}$ from $D+C$ by rectifiable crosscuts we obtain a simply-connected subdomain $D_{1}$ with $F$ on its boundary. Map $D_{1}$ onto $U_{x}:|x|<1$. Then $F$ is transformed to a closed set $F_{x}$ of positive linear measure on $\Gamma_{x}:|x|=1$ in virtue of Riesz's theorem ([9], [8]). The mapping of $D_{1}$ onto a subdomain $D_{\zeta}$ of $U_{\zeta}$ is one-to-one continuous, with their boundaries included. In the mapping $U_{x} \rightarrow D_{\zeta}$ the linear measure of the image $F_{\zeta}$ on $\Gamma_{\zeta}$ of $F_{x}$ is greater than $m\left(F_{x}\right)>0$ on account of the extension of Löwner's lemma (cf. [7], Chap. IV, §3), where $\zeta=0$ is supposed to correspond to $x=0$ without loss of generality. Accordingly $m\left(F_{\zeta}\right)>0$. Since $F_{\zeta}$ is contained in the angular image of $F$ on $E_{\zeta}$, the angular image on $E_{\zeta}$ of $E^{\prime} \supset F$ has a positive linear inner measure.

Once established this fact, the rest of the proof of our lemma is carried as follows. The function $\mu\left(\zeta, E_{\xi}\right)$ can be regarded as a one-valued bounded harmonic function in $U$. By Fatou's theorem it has angular limits a.e. on $T$. Denote the subset of $E$, where this function has angular limits less than 1 , by $E_{1}$, and its angular image on $E_{\xi}$ by $E_{\zeta}^{(1)}$. At every point of $E_{\zeta}^{(1)}$ there terminates a curve along which $\mu\left(\zeta, E_{\xi}\right)$ tends to a value $<1$, and so $\mu\left(\zeta, E_{\xi}\right)$ can not have the angular limit 1 at any point of $E_{\zeta}^{(1)}$. Hence the inner measure $\underline{m}\left(E_{\zeta}^{(1)}\right)=0$, because if $\underline{m}\left(E_{\zeta}^{(1)}\right)>0$ then $\mu\left(\zeta, E_{\zeta}\right)$ would have the angular limit 1 at a certain point of $E_{\xi}^{(1)} \subset E_{\dot{\jmath}}$. As we have seen that $\underline{m}\left(E_{\zeta}^{(1)}\right)>0$ follows from $m\left(E_{1}\right)>0$,

6) For its generalization, cf. [10] and [7], Chap. III, $\$ 2$. 
there must hold $m\left(E_{1}\right)=0$. Thus $\mu\left(\zeta, E_{\zeta}\right)$, which is considered as a function in $U$, has the radial limit 1 a.e. on $E$. Consequently we have $\mu(z, E) \leqq \mu\left(\zeta, E_{\zeta}\right)$.

Using this lemma the following theorem is proved:

Theorem 2. Suppose that $\underline{\Re}^{\text {ic }}$ is of hyperbolic type. Take a finite number of points $\left\{\underline{P}_{i}\right\}(i=1,2, \ldots, n)$ on $\mathfrak{R}$, remove from $\mathfrak{R}$ all the points lying over then and denote the remaining surface by $\widetilde{\mathfrak{H}}$. Then there holds

$$
\mu\left(P, \mathfrak{A}\left(\mathfrak{R}^{\infty}\right)\right)=\mu\left(P, \mathfrak{H}\left(\widetilde{\mathfrak{R}}^{\infty}\right)\right) .
$$

Proof. Map $\Re^{\infty}$ and $\widetilde{\Re}^{\infty}$ onto $U$ and $U_{\zeta}$, and let $E$ and $E_{\zeta}$ be the images on $\Gamma$ and $\Gamma_{\zeta}$ of $\mathfrak{U}\left(\Re^{\infty}\right)$ and $\mathfrak{U}\left(\widetilde{\mathfrak{H}}^{\infty}\right)$ respectively. Since $\mu\left(P, \mathfrak{H}\left(\mathfrak{R}^{\infty}\right)\right)=\mu(z, E)$ and $\mu\left(P, \mathfrak{R}\left(\left(\tilde{\Re}^{\infty}\right)\right)=\mu\left(\zeta, E_{\zeta}\right)\right.$, we want to prove $\mu(z, E)=\mu\left(\zeta, E_{\zeta}\right)$ at corresponding points. One inequality $\mu(z, E) \leqq \mu\left(\zeta, E_{\xi}\right)$ follows from the above lemma.

On the other hand, every radius terminating at a point on $E_{\zeta}$ is transformed to a curve in $U$ which terminates at a point of $E$ or at one of the inner points $\left\{z_{n}\right\}$ corresponding to $\left\{\underline{P}_{i}\right\}$. It is easily shown that $E$ coincides with the set of all such end-points on $T$. Since the number of $\left\{z_{n}\right\}$ is at most enumerably infinite, the part $E_{\zeta}^{\prime} \subset E_{\zeta}$ which corresponds to $\left\{z_{n}\right\}$ has linear measure zero. If $\zeta=0$ corresponds to $z=0, m\left(E_{\zeta}\right)=m\left(E_{\xi}-E_{\zeta}^{\prime}\right) \leqq m(E)$ on account of the extension of Löwner's lemma. Hence there follows the reverse inequality $\mu(z, E)$ $\geq \mu\left(\zeta, E_{\zeta}\right)$, and the required equality is obtained.

Let us now define the harmonic measure $\omega(P)$ of $\mathfrak{P}(\mathfrak{R})$ when ${\underline{\mathfrak{R}^{\prime}}}^{\infty}$ is not of hyperbolic type. Take one or two or three points on $\mathfrak{R}$ and remove from $\mathfrak{A}$ all the points lying over them so that the projection of the remaining surface $\widetilde{R}$ has a universal covering surface of hyperbolic type. We define harmonic measure $\omega(P)$ of $\mathfrak{H}(\Re)$ by $\mu\left(P, \mathfrak{H}\left(\widetilde{\mathfrak{H}}^{\infty}, \underline{\Re}\right)\right)$. Since every removed point of $\mathfrak{A}$ is isolated, $\omega(P)$ becomes harmonic everywhere on $\Re$. To avoid any possible ambiguity, we must, and shall, show that $\omega(P)$ is determined independently of the position of the points selected on $\stackrel{\Re}{\Omega}$.

Take a finite number of points on $\underline{\Omega}$ in another way, remove all the points lying over them from $\Re$ and $\widetilde{\Re}$, and denote the remaining surfaces by $\hat{\mathfrak{A}}$ and $\hat{\mathfrak{H}}$ respectively. The universal covering surface of the projection into $\Re$ of $\hat{\mathfrak{H}}$ is supposed to be of hyperbolic type here. On account of Theorem 2 we have

$$
\omega(P)=\mu\left(P, \mathfrak{H}\left(\widetilde{\mathfrak{R}}^{\infty}\right)\right)=\mu\left(P, \mathfrak{H}\left(\hat{\mathfrak{H}}^{\infty}\right)\right)=\mu\left(P, \mathfrak{H}\left(\hat{\mathfrak{R}}^{\infty}\right)\right) .
$$

Thus the harmonic measure $\omega(P)$ of $\mathfrak{U}(\Re)$ has been defined in all cases.

4. Prior to show a relation between $\omega(P)$ and the class $\left(B_{0}\right)$, we shall state some related results obtained recently.

Let $M$ be a covering surface over the $w$-plane, $K$ be a circular domain in the plane. and $\mathscr{D}$ be a domain of $\Re$, which lies over $K$ and whose boundary in $\Re$ 
lies over the boundary of $K$. Y. Nagai $[5]^{7)}$ and M. Tsuji [11] found independently that if $\mathscr{D}$ does not cover a set of positive capacity in $K$ then $\mathfrak{R}$ has a positive boundary, ${ }^{8)}$ and Y. Nagai [5] showed that, $n(w)$ denoting the number of points of $\Re$ lying over $w$, if the set $\{w ; n(w)<\sup n(w)\}$ is of positive capacity, then $K$ and $\mathscr{D}$ can be chosen such that $\mathscr{D}$ does not cover a set of positive capacity in $K$. Further map the universal covering surface of $\mathfrak{D}$ onto $U$ and denote the mapping function of $U$ into the $w$-plane by $f(z)$. A. Mori [4] proved the following theorem $: \Re \notin\left(B_{n}\right)$ if it does not arise that almost all radial limits of $f(z)$ lie on the boundary of $K$; and also showed that the requirement in this theorem is fulfilled if $D$ does not cover a set of positive capacity in $K$.

In this section we will prove

THEOREM 3. Let $\mathfrak{R}$ be a covering surface over an abstract Riemann surface $\mathfrak{R}$. If the harmonic measure $\omega(P)$ of the accessible boundary $\mathfrak{A}(\Re)$ is positive, then $\Re \notin\left(\mathrm{B}_{3}\right)$.

Proof. Without loss of generality we may suppose that $\underline{\Re}^{\infty}$ is of hyperbolic type. Let $\left\{\Im_{n}\right\}$ be a sequence of triangulations of $\mathfrak{R}$ such that $\Theta_{n+1}$ is a subdivision of $\Im_{n}$ and $\Im_{n}$ becomes as fine as we please when $n \rightarrow \infty$. We denote the triangles of $\Im_{n}$ by $\left\{\Delta_{i}^{(n)}\right\} \quad(i=1,2, \ldots \text {; finite or infinite })^{9}{ }^{\prime}$ Map $\mathfrak{R}^{\infty}$ onto $U$ and denote the function corresponding to $U \rightarrow \Re^{\infty} \rightarrow \mathfrak{R} \rightarrow \mathfrak{R}$ by $f(z)$. The set on $\Gamma$, where the radial limits of $f(z)$ lie in $\Delta_{i}^{(n)}$, will be denoted by $E_{i}^{(n)}$. Then every $E_{i}^{(n)}$ is linearly measurable and the image on $\Gamma$ of $\mathfrak{A}(\Re)$ is equal to $\sum_{i} E_{i}^{(n)}$ for each $n$. If there is such an $E_{i}^{(n)}$ as $0<m\left(E_{i}^{(n)}\right)<2 \pi$, its harmonic measure in $U$ is transformed into a one-valued non-constant harmonic function on $\Re$. Thus the required function is obtained.

On the contrary, suppose that for every $n$ there existed $i(n)$ such that $m\left(E_{i(n)}^{(n)}\right)=2 \pi$. Then $E_{i(n)}^{(n)} \supset E_{i(n+1)}^{(n+1)}$ and $\Delta_{i(n)}^{(n)} \supset \Delta_{i(n+1)}^{(n+1)}$. If we compose a nonconstant meromorphic function $\mathscr{D}(\underline{P})$ on $\underline{\Re}$ and $f(z)$, the angular limits of the composed function $F(z)$ would be equal to one and the same value $\mathscr{Q}\left(\bigcap_{n=1}^{\infty} \Delta_{i(n)}^{(n)}\right)$ at every point of $\bigcap_{n=1}^{\infty} E_{i(n)}^{(n)}$ with $m\left(\bigcap_{n=1}^{\infty} E_{i(n)}^{(n)}\right)=2 \pi$. On account of Lusin-Priwaloff's theorem $F(z)$ would be a constant and this is a contradiction, which completes the proof.

THEOREM 4. Let $\mathfrak{R}$ be a covering surface over an abstract Riemann surface 奋. If $\Re$ does not cover a set of positive capacity on $\underline{R}^{10)}$ then $\omega(P)>0$.

7) His statement is of a slightly different form.

8) As is known, a Green's function exists on $\mathfrak{R}$ if and only if $\Re$ has a positive boundary. Cf. [7], Chap. II, $\$ 4$.

9) $\left\{\Delta_{i}^{(n)}\right\}$ are made half open so that they are mutually disjoint for every fixed $n$.

10) This means that the image in a parameter circle, corresponding to a certain neighborhood on $\mathbb{R}$, is of positive capacity. 
Proof. First suppose that $\underline{\Re}^{\infty}$ is of hyperbolic type, and map $\Re^{\infty}$ and $\underline{\Re}^{\infty}$ onto $U$ and $U_{w}:|w|<1$ respectively. Any branch of the function corresponding to $U \rightarrow \Re^{\infty} \rightarrow \mathfrak{R}^{\infty} \rightarrow U_{w}$ will be denoted by $w=F(z)$. $F(z)$ does not take values of a set of positive capacity in $U_{w}$ and the image $E$ on $I$ of $\mathfrak{H}(\mathfrak{R})$ coincides with the place where $F(z)$ has limits lying inside $U_{w}$. Hence by Frostman's theorem [2] for functions of class $(U), m(E)>0$. Thus $\omega(P)=\mu(z, E)>0$. The case when $\mathfrak{R}^{\infty}$ is not of hyperbolic type is now easily treated.

Corollary. Let $\mathfrak{D}$ and $\mathfrak{D}$ be domains of $\mathfrak{R}$ and $\mathfrak{R}$ respectively such that $\mathscr{D}$ lies over $\mathfrak{D}$ and the boundary of $\mathscr{D}$ in $\Re$ does not lie over the inside of $\mathfrak{D}$. If $\mathfrak{D}$ does not cover a set of positive capacity in $\mathbb{D}$ then $\omega(P)$ of $\mathfrak{H}(\mathfrak{R})$ is positive.

For, the harmonic measure of $\mathfrak{A}(\mathfrak{D}, \mathfrak{D})$ is positive by Theorem 4 . On account of the extension of Löwner's lemma $\omega(P)$ of $\mathfrak{H}(\mathfrak{R}, \underline{\mathfrak{R}})$ is greater than it and hence is positive.

5. Theorem 3 is trivial when $\omega(P)$ is not a constant, and is interesting only when $\omega(P) \equiv 1$.

THEOREM 5. Let $\mathfrak{R}$ be a covering surface of F-type over $\mathfrak{R}$. Then $\omega(P) \equiv 1$.

Proof. If $\underline{\Re}^{\infty}$ is of hyperbolic type, $\omega(P)=\mu(z, E) \equiv 1$ by Theorem 1 , where $E$ is the image on $\Gamma$ of $\mathfrak{A}(\mathfrak{R})$.

In the case when $\underline{\Re}^{\prime \infty}$ is not so, define $\widetilde{\mathfrak{R}}$ as in $\S 3$ and map $\widetilde{\mathfrak{R}}^{\infty}$ onto $U_{\zeta}:|\zeta|$ $<1$. We shall denote the image on $|\zeta|=1$ of $\mathfrak{A}(\widetilde{\Re})$ by $E_{\xi}$, and the set on $\Gamma$, where the mapping function of $U$ into $\Re$ has angular limits, by $E$. Then by Lemma in $\S 3$ there follows $\mu(z, E) \leqq \mu\left(\zeta, E_{\zeta}\right)$ at corresponding points. Since $m(E)=2 \pi$, we have $\omega(P)=\mu\left(\zeta, E_{\zeta}\right)=\mu(z, E) \equiv 1$.

We next give a condition under which $\mathfrak{R}$ becomes of F-type, by

THEOREM 6. (Extension of Theorem 3.3 in [7] .) Let $\mathfrak{R}$ be a covering surface over an abstract Riemann surface $\mathfrak{R}$ such that the projection of $\Re$ is com-

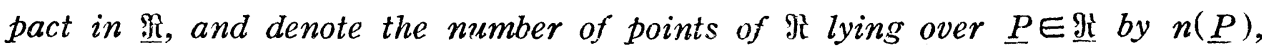
computing the multiplicity at each branch point of $\Re$. If the set $\underline{E}=\{\underline{P} \in \underline{\Re}$; $n(\underline{P})<N=\sup n(\underline{P})\}$ is of positive capacity on $\underline{R}$, then $\Re$ is of F-type.

Proof. The set $E_{k}=\{\underline{P} ; n(\underline{P}) \leqq k\}$ is a closed set for each $k$. Since $\underline{E}$ $=\bigcup_{0 \leqq k} \underset{<N}{E_{k}}$ and is of positive capacity, there exitst the smallest number $k_{0}$ for which $E_{k_{0}}$ is of positive capacity. If $k_{0}=0$ there follows $\Re \notin\left(\mathrm{B}_{0}\right)$ from Theorems 4 and 3. The set $\underline{E}_{k_{0}}^{b}-\underline{E}_{k_{0}}^{b} \cap \underline{E}_{k_{0}-1}$ for $k_{0}>0$ is also of positive capacity, where $E_{k_{0}}^{b}$ denotes the boundary in $\underline{\Re}$ of $\underline{E}_{k_{0}}^{b}$. Let $\underline{P}_{0}$ be an arbitrary point of its transfinite kernel. There lie $l \leqq k_{0}$ points of $\Re: P_{1}, P_{2}, \ldots, P_{l}$, over $\underline{P}_{0}$. Over a sufficiently small neighborhood $\underline{N}$ on $\underline{? R}$ of $\underline{P}_{0}$ there exists another connected piece $\mathfrak{D}$ of $\mathfrak{R}$ than those containing $\left\{P_{j}\right\}(1 \leqq j \leqq l)$. Since this domain 
D does not cover a set of positive capacity in $\underline{N}, \omega(P)>0$ by Corollary of Theorem 4 and hence $\mathfrak{R} \notin\left(\mathrm{B}_{0}\right)$ by Theorem $3 .^{11)}$ Thus $\mathfrak{R}$ has a positive boundary.

Map $\mathfrak{R}^{\infty}$, which is of hyperbolic type, onto $U$, and consider a Green's function $G(P)$ on $\Re$ as a function in $U$. The angular limit of $G(P(z))$ is equal to 1 at every point of a set $G_{z}$ of linear measure $2 \pi$ (cf. [6], Chap. VII). In a similar manner as in the proof of Lemma in $\S 3$, we get a domain $D$ in $U$ such that it contains an end-part of the angular domain: $\left|\arg \left(1-e^{-i \theta} z\right)\right|<\frac{\pi}{2}-\frac{1}{p}$ $(>0)$ at every point $e^{i \theta}$ of a closed set $F_{n} \subset G_{z}$ with $m\left(F_{n}\right)>2 \pi-\frac{1}{n}$ and is bounded by a rectifiable curve $C$ and $G(P(z)) \rightarrow 0$ uniformly as $z \rightarrow F_{n}$ from the inside of $D$. Since $G\left(P_{j}\right)>0(1 \leqq j \leqq l)$, the image of $\left\{P_{j}\right\}$ in $D$ or on $C$ consists of a finite number of points. We remove these points from $D+C$ by rectifiable cross-cuts such that the remaining domain $D_{1}$ is simply-connected and $F_{n}$ lies on its boundary. Map $D_{1}$ onto $U_{\zeta}:|\zeta|<1$ and consider in $U_{\zeta}$ the function $f(z)$ which maps $U$ into $\underline{R}$. Since the image on $\Re$ of $D_{1}$ dose not contain points near $\left\{P_{j}\right\}$, it does not cover a set of positive capacity on $\underline{R}$. Hence by Theorem 3.3 in [7] $f(z(\zeta))$ has angular limits a.e. on $\Gamma_{\zeta}:|\zeta|=1$.

Now we denote the angular domain: $\left|\arg \left(1-e^{-i \theta} z\right)\right|<\frac{\pi}{2}-\frac{2}{p}$ at $e^{i \theta}$ by $A_{b}(\theta)$. By the method in proving the angular proportionality at boundary points in conformal mapping (cf. [1]), we can show that an end-part of $A_{\phi}(\theta)$ at $e^{i \theta} \in F_{n}$ is transformed to a domain inside an angular domain at $\zeta\left(e^{i \theta}\right)$ when $D_{1}$ is mapped onto $U_{\zeta}$. Thus $f(z)$ has a limit from the inside of $A_{p}(\theta)$ at the image $e^{i \theta}$ of a point on $\Gamma_{\zeta}$ where $f(z(\zeta))$ has an angular limit. By Riesz's theorem the image on $\Gamma$ of any null set on $\Gamma_{\zeta}$ is a null set. Therefore $f(z)$ has a limit from the inside of $A_{p}(\theta)$ at every point $e^{i \theta}$ of a set of measure $2 \pi-\frac{1}{n}$. By letting $n \rightarrow \infty$ we see that $f^{\prime}(z)$ has limits everywhere on $\Gamma$ from the inside of $A_{p}(\theta)$, except on a set $H_{p}$ with $m\left(H_{p}\right)=0$. Hence $f(z)$ has an angular limit at every point of $\Gamma-\bigcup_{p=1}^{\infty} H_{p}$. Since $m\left(\bigcup_{p=1}^{\infty} H_{p}\right)=0, f(z)$ has an angular limit a.e. on $T$. Thus $\mathfrak{R}$ is of $F$-type.

6. In the following we shall see some relations between various concepts defined in this note, under the assumption that $\mathfrak{R}^{\prime}$ is not of hyperbolic type; if this is of hyperbolic type the relations are stated in simpler forms.

First we supose that $\Re$ has a null boundary. The surface $\widetilde{\Re}$ which is defined in $\S 3$ has also a null boundary by Lemma 1.3 in [7]. Since no bounded and non-constant continuous superharmonic function exists on a surface with null boundary by Lemma 1.2 in [7], the upper classes $\mathfrak{B}(\mathfrak{H})$ and $\mathfrak{B}(\widetilde{\mathfrak{P}})$ contain merely the constant 1 . Thus $\mu(P, \mathfrak{H}(\mathfrak{R}))=\mu(P, \mathfrak{A}(\widetilde{\mathfrak{R}})) \equiv 1$. On the other hand

11) Here we see that Theorem 6 does not serve as an example of the application of the fact, which follows from Theorems 5 and 3 , that $\Re$ of $F$-type does not belong to $\left(B_{0}\right)$. 
Theorem 3 shows that $\omega(P)=\mu(P).\left(\left(\widetilde{\Re}^{\infty}\right)\right) \equiv 0$. If $\Re^{\infty}$ is of parabclic type, this has a null boundary and hence $\mu\left(P, \mathfrak{M}\left(\Re^{\infty}\right)\right) \equiv 1$. We shall show that $\mu\left(P, \mathfrak{M}\left(\Re^{\infty}\right)\right)$ $\equiv 0$ if $\mathfrak{R}^{\approx}$ is of hyperbolic type. Any curve determining an A.B.P. of $\mathfrak{R}$ converges to an ideal boundary component of $\Re^{12}{ }^{12} \mathrm{M}$. Tsuji [11] showred that the image $E_{0}$ on $\Gamma$ of the ideal boundary of $\Re$ has linear measure zero in the mapping of $\mathfrak{R}^{*}$ onto $U$. Hence any image of a determining curve of an A.B.P. terminates at a point of $E_{0}$, and the lower cover of the class consisting of all the non-negative continuous superharmonic functions $\{v(z)\}$ not greater than 1 and with $\lim _{z \rightarrow F_{0}} v(z)=1$ is zero. For any $\varepsilon>0$ and an arbitrary point $z_{0}$, we can find in this class a function $v_{0}(z)$ with $v_{0}\left(z_{0}\right)<\varepsilon_{\text {. If }} v_{0}(z)$ is regarded as a function on $\mathfrak{R}^{\infty}$, it belongs to $\mathfrak{B}\left(\mathfrak{A}^{\infty}\right)$. By the arbitrarinesses of $z_{0}$ and $\varepsilon$, the lower cover $\mu\left(P, \mathfrak{A}\left(\mathfrak{H}^{\Re}\right)\right)$ of $\mathfrak{B}\left(\mathfrak{H}^{\infty}\right)$ is zero constantly.

Let us now pass to the case where $\mathfrak{R}$ has a positive boundary. Set $\mathfrak{R}-\widetilde{\mathfrak{R}}$ $=\left\{P_{n}\right\}$ and let $G_{n}(P)$ be the Green's function on $\Re$ with its pole at $P_{n}$. For an arbitrary point $P_{0} \in \widetilde{\Re}$, the function $g(P)=\sum_{n} \frac{1}{n^{2}} \cdot G_{n}(P)$ represents a harmonic function on $\widetilde{\Re}$ in virtue of Harnack's theorem. For any $\varepsilon>0$ and $v(P)$ $\in \mathfrak{B}(\mathfrak{H}), \min (1, v(P)+\varepsilon g(P))$ belongs to $\mathfrak{B}(\widetilde{\mathfrak{R}})$ if it is considered as a function on $\widetilde{\mathfrak{R}}$. $\varepsilon$ and $v(P)$ being arbitrary, there follows $\mu(P, \mathfrak{A}(\mathfrak{R})) \geqslant \mu(P, \mathfrak{A}(\widetilde{\mathfrak{R}}))$. Conversely any $v(P) \in \mathfrak{B}(\widetilde{\Re})$ belongs to $\mathfrak{B}(\mathfrak{R})$ if the value 1 is supplemented to $v(P)$ at $\mathfrak{R}-\widetilde{\mathfrak{R}}$. Hence $\mu(P, \mathfrak{A}(\widetilde{\mathfrak{A}})) \Longrightarrow \mu(P, \mathfrak{A}(\mathfrak{R}))$ and the equality follows. Further there holds $\mu(P, \mathfrak{F}(\mathfrak{R})) \geqq \mu\left(P, \mathfrak{A}\left(\mathfrak{R}^{\infty}\right)\right)$, because any $v(P) \in \mathfrak{B}(\mathfrak{R})$ considered on $\mathfrak{R}^{\infty}$ belongs to $\mathfrak{B}\left(\Re^{\infty}\right)$. It is yet unknown whether there is or not a case when a proper inequality holds. Since, for any $v(P) \in \mathfrak{B}\left(\mathfrak{H}^{\infty}\right)$ and $\varepsilon>0$, $\min (1$, $v(P)+\varepsilon g(P)) \in \mathfrak{B}\left(\widetilde{\mathfrak{A}}^{\infty}\right)$. we can conclude the inequality $\mu\left(P, \mathfrak{A}\left(\mathfrak{R}^{\infty}\right)\right) \geqslant \mu(P$. $\left.\mathfrak{Y}\left(\widetilde{\mathfrak{R}}^{\infty}\right)\right)$. At present we have no example in which the inequality of this relation is proper. The relations are summarized in

$$
\mu(P, \mathfrak{P}(\mathfrak{\Re}))=\mu(P . \mathfrak{H}(\widetilde{\mathfrak{R}})) \geq \mu\left(P, \mathfrak{H}\left(\mathfrak{R}^{\infty}\right)\right) \geq \mu\left(P, \mathfrak{H}\left(\widetilde{\mathfrak{R}}^{\infty}\right)\right) .
$$

Generalizing the definition in [7], Chap. IV, $\$ 2$, we will say that a covering surface $\Re$ with positive boundary over $\mathfrak{R}$ is of $\mathrm{D}$-type (relatively to $\mathfrak{R}$ ), if any upper bounded continuous subharmonic function $u(P)$ is non-positive whenever $\lim u(P) \leqq 0$ as $P \rightarrow \mathfrak{T}(\Re)$ along every determining curve of an A.B.P. Since, for any $v(P) \in \mathfrak{B}(\Re), 1-v(P)$ may be taken as above $u(P)$ and conversely. for any such a $u(P)<M(>0)$. $\min (1,1-u(P) / M) \in \mathfrak{B}(\mathfrak{R})$, we find that $\mathfrak{R}$ is of D-type if and only if $\mu(P . \mathfrak{A}(\mathfrak{R})) \equiv 1$. Taking Theorem 4.1 in [7] into account. for $\mathfrak{R}$ with positive boundary we can write

$$
\begin{array}{cc}
\mu(P, \mathfrak{N}(\Re)) \equiv 1 & \leftarrow \text { D-type } \\
\downarrow & \stackrel{ \pm}{I} \\
\omega(P) \equiv 1 & \leftarrow \text { F-type },
\end{array}
$$

12) For the definition of an ideal boundary component, cf. [7]. Chap. III, $\$ 5$. 
where I means that this is known to us only in a special case. Theorem 4.2 in [7] is included in this scheme. Here are left some questions open still.

\section{BIBLIOGRAPHY}

[1] C. Carathéodory: Elementare Beweis für den Fundamentalsatz der konformen Abbildung, Schwarz Festschrift, Berlin (1914), pp. 19-41.

[2] O. Frostman: Potentiel d'équilibre et capacité des ensembles avec quelques applications à la théorie des fonctions, Meddel. Lunds. Univ. Mat. Sem., 3 (1935), pp. 1-118.

[3] N. Lusin and J. Priwaloff: Sur l'unicité et la multiplicité des fonctions analytiques, Ann. École Norm., 42 (1925), pp. 143-191.

[4] A. Mori: On Riemann surfaces, on which no bounded harmonic function exists, which will appear in Journ. Math. Soc. Japan.

[5] Y. Nagai: On the behaviour of the boundary of Riemann surfaces, II, Proc. Japan Acad., 26 (1950), pp. 10-16 (of No. 6).

[6] R. Nevanlinna: Eindeutige analytische Funktionen, Berlin (1936).

[7] M. Ohtsuka: Dirichlet problems on Riemann surfaces and conformal mappings, Nagoya Math. Journ., 3 (1951), pp. 91-137.

[8] F. Riesz: Über die Randwerte einer analytischen Funktion, Math. Z., 18 (1923), pp. 8795.

[9] F. and M. Riesz: Über die Randwerte einer analytischen Funktion, 4 Congrès Scand. Stockholm, (1916), pp. 27-44.

[10] M. Tsuji: Theory of meromorphic function in a neighbourhood of a closed set of capacity zero, Jap. J. Math., 19 (1944), pp. 139-154.

[11] M. Tsuji: Some metrical theorems on Fuchsian groups, Kōdai Math. Sem. Report, Nos. 4-5 (1950), pp. 89-93.

[12] K. I. Virtanen: Über die Existenz von beschränkten harmonischen Funktionen auf offenen Riemannschen Flächen, Ann. Acad. Sci. Fenn., A. I., (1950), No. 75, 7 pp.

Mathematical Institute, Nagoya University 